
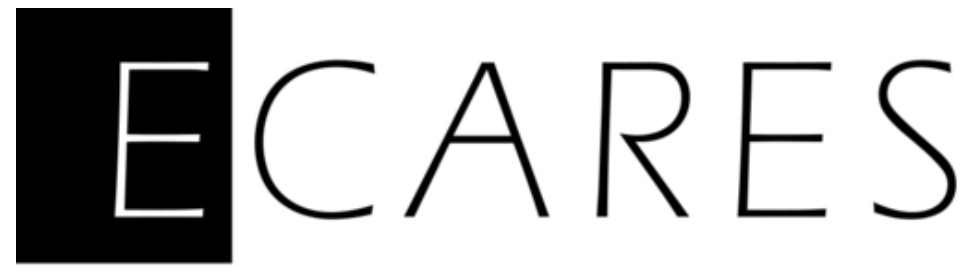

\title{
Power Method Tâtonnements for Cobb-Douglas Economies
}

\author{
V. Shikhman \\ Mathematics Department, Technische Universität Chemnitz \\ Yu. Nesterov \\ CORE, Université catholique de Louvain \\ V. Ginsburgh \\ ECARES, Université libre de Bruxelles and CORE, Université catholique de Louvain
}

March 2017

ECARES working paper 2017-09 


\title{
Power Method Tâtonnements for Cobb-Douglas Economies
}

\author{
V. Shikhman * Yu. Nesterov ${ }^{\dagger} \quad$ V. Ginsburgh ${ }^{\ddagger}$ \\ March 15, 2017
}

\begin{abstract}
We consider an economy with consumers maximizing Cobb-Douglas utilities from the algorithmic perspective. It is known that in this case finding equilibrium prices reduces to the eigenvalue problem for a particularly structured stochastic matrix. We show that the power method for solving this eigenvalue problem can be naturally interpreted as a tâtonnement executed by an auctioneer. Its linear rate of convergence is established under the reasonable assumption of pairwise connectivity w.r.t. commodities within submarkets. We show that the pairwise connectivity remains valid under sufficiently small perturbations of consumers' tastes and endowments. Moreover, the property of pairwise connectivity holds for almost all Cobb-Douglas economies.
\end{abstract}

Keywords: exchange economy, Cobb-Douglas utility, tâtonnement, power method, stochastic matrix, regular economy

${ }^{*}$ Department of Mathematics, Technische Universität Chemnitz, Reichenhainer Str. 41, 09126 Chemnitz, Germany; e-mail: vladimir.shikhman@mathematik.tu-chemnitz.de.

${ }^{\dagger}$ Center for Operations Research and Econometrics (CORE), Catholic University of Louvain (UCL), 34 voie du Roman Pays, 1348 Louvain-la-Neuve, Belgium; e-mail: yurii.nesterov@uclouvain.be.

${ }^{\ddagger}$ ECARES, Universite Libre de Bruxelles, Avenue F. D. Roosevelt 50, CP 114, B-1050 Brussels, Belgium and CORE 34 voie du Roman Pays, 1348 Louvain-la-Neuve, Belgium; e-mail: vginsburg@ulb.ac.be.

The research of the second author has been supported by a grant "Action de recherche concertè ARC 04/09-315" from the "Direction de la recherche scientifique - Communautè française de Belgique". 


\section{Introduction}

Starting with Walras in 1874, price tâtonnement methods, in which the price of each commodity is adjusted to it excess demand, have a long story in solving competitive equilibrium models, a story with ups and downs, with hopes and disappointments. Arrow and Hurwicz (1958) and Arrow et al. (1959) give particular conditions under which equilibrium is unique and tâtonnement is stable (in particular, if there is no trade in equilibrium, or if the excess demand function satisfies gross substitutability, diagonal dominance, or the weak axiom of revealed preference, along the path of the tâtonnement equation). To show this, they exhibit a Lyapunov function for each situation, which ensures convergence of the process.

One year later Scarf (1960) constructed examples with three commodities and three consumers showing that even if the economy has only one equilibrium, tâtonnement could not be used to find it, ruining the hope that tâtonnement would work in many cases. This led Scarf (1967) and Scarf (1973) to discover algorithms based on Brouwer's and Kakutani's fixed point theorems that could find (approximate) equilibria.

Eaves (1976) devises a finite algorithm for the exchange model with linear utilities, and Eaves (1985) describes a method for the exchange model with Cobb-Douglas utilities, which leads to an equilibrium in a finite number of approximately $n^{3} / 3+n^{2} m$ multiplications and additions, where $m$ and $n$ are respectively the numbers of consumers and commodities. Note that Eaves' method needs explicit knowledge of consumers' tastes and endowments is needed.

In this paper, we reconsider the same Cobb-Douglas model as Eaves (1985) who showed that solving for equilibrium reduces to the eigenvector problem for a specially structured stochastic matrix. We discuss two tâtonnements which approximate equilibria with guaranteed worst-case efficiency. Roughly speaking, the auctioneer sets new prices to be proportional to the value of aggregate demand measured in old prices. This natural tâtonnement can be viewed as the well-known power method for finding stationary states of Markov chains. Applying recent results by Nesterov and Nemirovski (2015) on finding stationary states of Markov chains, we get insights on how economic equilibria can easily be computed in reasonable time. In tâtonnements based on the power method, the auctioneerdoes not need information on consumers' tastes and endowments, but only aggregate demand. Another complementary line of research has been recently suggested by $\mathrm{Du}$ et al. (2015) on how to obtain Google rankings by using market mechanisms.

The paper is organized as follows. Section 2 describes the competitive equilibrium model with Cobb-Douglas utilities, derives the demand functions and introduces a property of symmetric access that ensures existence of at least one equilibrium. Section 3 is devoted to two tâtonnement algorithms - with and without averaging. The averaging scheme converges not faster than sublinearly, the other may eventually diverge. If the additional pairwise connectivity assumption is also satisfied, then tâtonnement with averaging converges at sublinear rate, without averaging - at the linear rate. Sufficient conditions for pairwise connectivity are given, and economic examples involving capital, labor, money, and taxation are discussed. In Section 4 we define regular Cobb-Douglas economies as having symmetric access, no submarkets, and being pairwise connected. Regularity of Cobb-Douglas economies is shown to be stable w.r.t. sufficiently small perturbations of consumers' tastes and endowments. Moreover, almost all (w.r.t. Lebesgue 
measure) Cobb-Douglas economies are proven to be regular. This result is of the same topological spirit widely propagated within the general equilibrium theory e.g. by Debreu (1970), Mas-Collel (1985), Bonnisseau et al. (2001), and Bonnisseau (2003).

Notation. We denote by $\mathbb{R}^{n}$ the space of $n$-dimensional column vectors

$$
x=\left(x^{(1)}, \ldots, x^{(n)}\right)^{T},
$$

and by $\mathbb{R}_{+}^{n}$ the set of all vectors with nonnegative components. For $x \in \mathbb{R}^{n}$ we also write $x \geq 0$ or $x>0$ to express that all components of $x$ are nonnegative or positive, respectively. The $j$-th coordinate vector is denoted by $e_{j}$, and the vector of all ones by $e$. For $x, y \in \mathbb{R}^{n}$, we introduce the standard scalar product and the Hadamard product

$$
x y=\sum_{j=1}^{n} x^{(j)} y^{(j)}, \quad x \circ y=\left(x^{(j)} y^{(j)}\right)_{j=1}^{n} \in \mathbb{R}^{n} .
$$

For $x=\left(x^{(1)}, \ldots, x^{(n)}\right)^{T} \in \mathbb{R}^{n}$ we denote

$$
\frac{1}{x}=\left(\frac{1}{x^{(1)}}, \ldots, \frac{1}{x^{(n)}}\right)^{T} \text { if } x^{(j)} \neq 0, \quad j=1, \ldots, n .
$$

Given $v=\left(v^{(1)}, \ldots, v^{(n)}\right)^{T}>0$ from $\mathbb{R}^{n}$, we use the following scaled 1-norm:

$$
\|x\|_{v}=\sum_{j=1}^{n} v^{(j)}\left|x^{(j)}\right| .
$$

For a vector $v>0$ from $\mathbb{R}^{n}$ and a positive number $t>0$ we define the interior of the scaled simplex as

$$
\Delta_{t}(v):=\left\{x \in \mathbb{R}^{n} \mid x v=t, \quad x>0\right\} .
$$

For a $n \times n$ real matrix $A$ with nonnegative elements we write $A \geq 0$.

\section{Exchange economy with Cobb-Douglas utilities}

Let $m$ consumers denoted by $i=1, \ldots, m$ exchange commodities on $n$ markets denoted by $j=1, \ldots, n$. Consumer $i$ 's utility function is:

$$
u_{i}\left(x_{i}\right):=\beta_{i} \prod_{j=1}^{n}\left(x_{i}^{(j)}\right)^{\alpha_{i j}},
$$

where $\beta_{i} \in \mathbb{R}_{+}$is a scaling coefficient, and $\alpha_{i j} \in \mathbb{R}_{+}$is the elasticity w.r.t. commodity $j$ of $i$-th consumer's utility. We denote the vectors of elasticities as $\alpha_{i}:=\left(\alpha_{i j}\right)_{j=1}^{n}$. Consumer $i$ has endowment $\omega_{i}:=\left(\omega_{i}^{(1)}, \ldots, \omega_{i}^{(n)}\right)^{T} \in \mathbb{R}_{+}^{n}, \omega_{i} \neq 0 .{ }^{1}$ The overall endowment is $\omega:=\sum_{i=1}^{m} \omega_{i}>0 .^{2}$

\footnotetext{
${ }^{1}$ If $\omega_{i}=0$, we exclude this consumer from our economy.

${ }^{2}$ If one of the commodities has zero total initial endowment, we exclude it from the model.
} 
Definition 1 (Exchange equilibrium) The vector of prices and consumption quantities $\left(p^{*},\left(x_{i}^{*}\right)_{i=1}^{m}\right)$ is an exchange equilibrium, if

(i) consumers maximize Cobb-Douglas utilities w.r.t. budget constraints, i.e.

$$
x_{i}^{*} \in \arg \max _{x_{i} \geq 0}\left\{u_{i}\left(x_{i}\right) \mid p^{*} x_{i} \leq p^{*} \omega_{i}\right\}, \quad i=1, \ldots, m,
$$

(ii) the market clearing condition holds, i.e.

$$
p^{*} \geq 0, \quad z\left(p^{*}\right)=\sum_{i=1}^{m} x_{i}^{*}-\omega \leq 0, \quad p^{*} z\left(p^{*}\right)=0 .
$$

In these expressions $z\left(p^{*}\right)$ is the excess demand vector evaluated at prices $p^{*}$, and $p^{*} z\left(p^{*}\right)=0$ is the Walras Law.

We now make the two following additional assumptions:

Assumption 1 (Cobb-Douglas elasticities I) Each commodity $j$ is desired by some consumer $i$, i.e. for each commodity $j$ there exists a consumer $i$ with $\alpha_{i j}>0$.

Assumption 2 (Cobb-Douglas elasticities II) Each consumer $i$ desires some commodity $j$, i.e. for each consumer $i$ there exists a commodity $j$ with $\alpha_{i j}>0$.

Let Assumptions 1 and 2 hold throughout this Section 2. Assumption 1 ensures that in every equilibrium, $p^{*}>0$ and $z(p *)=0$. Otherwise some consumer would purchase infinite amounts of the zero priced commodity. Assumption 2 ensures that $\sum_{j=1}^{n} \alpha_{i j}>0$ for all $i$ and that an exchange equilibrium is preserved under a normalization of elasticities, i. e. for Cobb-Douglas utility functions

$$
v_{i}\left(x_{i}\right):=\beta_{i} \prod_{j=1}^{n}\left(x_{i}^{(j)}\right)^{\frac{\alpha_{i j}}{\sum_{j=1}^{n} \alpha_{i j}}} .
$$

Hence, we may, without loss of generality, assume that $\sum_{j=1}^{n} \alpha_{i j}=1$ for all $i$.

For positive prices $p>0$ we consider the $i$-th consumer's utility maximization problem

$$
\max _{x_{i} \geq 0}\left\{u_{i}\left(x_{i}\right) \mid p x_{i} \leq p \omega_{i}\right\} .
$$

Due to Assumption 2, each consumer will spend his entire budget, i. e. the Walras Law will hold for any price vector $p>0$. Given these prices, consumer $i$ 's demands are

$$
x_{i}^{(j)}(p)=\frac{\alpha_{i j}}{p^{(j)}} p \omega_{i}, \quad j=1, \ldots, n .
$$

We denote the aggregate demand by

$$
x(p):=\sum_{i=1}^{m} x_{i}(p)
$$


The market clearing condition $z(p)=0$ now reads

$$
\sum_{i=1}^{m} \alpha_{i j} p \omega_{i}-p^{(j)} \omega^{(j)}=0, \quad j=1, \ldots, n
$$

or, equivalently,

$$
\frac{1}{\omega^{(j)}} \sum_{i=1}^{m} \alpha_{i j} \omega_{i} p=p^{(j)}, \quad j=1, \ldots, n .
$$

In matrix form we get the eigenvector problem

$$
A p=p,
$$

where $A$ is the matrix obtained by the following row-wise setting

$$
A:=\left(\begin{array}{c}
\frac{1}{\omega^{(1)}} \sum_{i=1}^{m} \alpha_{i 1} \omega_{i}^{T} \\
\vdots \\
\frac{1}{\omega^{(n)}} \sum_{i=1}^{m} \alpha_{i n} \omega_{i}^{T}
\end{array}\right) .
$$

Matrix A contains obviously nonnegative elements, i.e. $A \geq 0$. Moreover, $\omega^{T} A=\omega^{T}$, since $\sum_{j=1}^{n} \alpha_{i j}=1$. Up to a scaling of coordinates with $\omega^{(1)}, \ldots, \omega^{(n)}$ in the image space, the characteristic matrix $A$ is column-stochastic. Therefore, the theory of stochastic matrices applies.

Recall that an $n \times n$ matrix $B \geq 0$ is called column-stochastic if $e^{T} B=e^{T}$. An $n \times n$ matrix $B \geq 0$ is called irreducible if there exists no permutation matrix $P$ such that

$$
P^{-1} B P=\left(\begin{array}{cc}
C & 0 \\
D & E
\end{array}\right)
$$

where $C$ and $E$ are square matrices of a dimension smaller than $n$. An $n \times n$ matrix $B \geq 0$ is called completely reducible if it is a direct sum of irreducible square $n_{r} \times n_{r}$ matrices $B_{r}, r=1, \ldots, R$, i.e. there exists a permutation matrix $P$ with

$$
P^{-1} B P=\left(\begin{array}{cccc}
B_{1} & 0 & \ldots & 0 \\
0 & B_{2} & \ldots & 0 \\
\ldots & \ldots & \ldots & \ldots \\
0 & 0 & \ldots & B_{R}
\end{array}\right)
$$

This representation of a completely reducible matrix $B$ is unique up to permutations of the coordinates within each diagonal block and up to the order of $B_{1}, \ldots, B_{R}$.

Theorem 1 (Complete reducibility, Schaefer (1974)) For a column-stochastic matrix $B \geq 0$, the following assertions are equivalent:

(a) $B$ is completely reducible,

(b) B possesses a positive eigenvector for eigenvalue 1,

(c) $x \geq 0, x \neq 0$ implies $x^{T} P \geq 0, x^{T} P \neq 0$, where $P=\lim _{k \rightarrow \infty} \frac{I+B+B^{2}+\cdots+B^{k}}{k+1}$. 
Complete reducibility of the matrix $A$ can be equivalently expressed in economic terms. Here, we follow Eaves (1985) and define the property of symmetric access:

Definition 2 (Symmetric access, Eaves (1985)) Given two different commodities $k$ and $l$, commodity $k$ is defined to access commodity $l$ if there is a sequence of commodities $l=j_{1} \ldots, j_{S+1}=k$ such that the $\left(j_{s}, j_{s+1}\right)$-th element of $A$ is positive, i.e.

$$
\frac{1}{\omega^{\left(j_{s}\right)}} \sum_{i=1}^{m} \alpha_{i j_{s}} \omega_{i}^{\left(j_{s+1}\right)}>0, \quad s=1, \ldots, S .
$$

That is, there is a sequence of consumers $i_{1}, \ldots, i_{S}$, where consumer $i_{s}$ both possesses commodity $j_{s+1}$ (so that $\omega_{i_{S}}^{\left(j_{s+1}\right)}>0$ ), and desires commodity $j_{s}$ (so that $\alpha_{i_{S} j_{s}}>0$ ), for $s=1, \ldots, S$. A Cobb-Douglas economy is said to have symmetric access if for every pair of different commodities either they access each other or neither accesses the other.

Symmetric access of a Cobb-Douglas economy ensures that it can be completely decomposed into submarkets. In fact, since $A \geq 0$ is completely reducible, it can be represented as a direct sum of irreducible square $n_{r} \times n_{r}$ matrices $A_{r}, r=1, \ldots, R$. Up to a permutation of commodities, we may write w.l.o.g.

$$
A=\left(\begin{array}{cccc}
A_{1} & 0 & \ldots & 0 \\
0 & A_{2} & \ldots & 0 \\
\ldots & \ldots & \ldots & \ldots \\
0 & 0 & \ldots & A_{R}
\end{array}\right)
$$

The $n_{r}$ commodities corresponding to $A_{r}$ represent the $r$-th submarket of the CobbDouglas economy. We denote by $N_{r} \subset\{1, \ldots, n\}$ the set of $r$-th submarket's commodities. Note that every two commodities within one and the same submarket access each other, whereas every two commodities from different submarkets do not. Moreover, each consumer cannot posses commodities from one submarket and desire commodities from another. Hence, submarkets are no longer connected with each other through consumers' tastes and endowments. ${ }^{3}$ The total number of submarkets is $R$. If $R=1$ we say that the corresponding Cobb-Douglas economy has no submarkets.

As shown by Eaves (1985), a Cobb-Douglas economy has symmetric access if and only if its corresponding matrix $A$ is completely reducible. Hence, this is equivalent to the existence of a not necessarily unique equilibrium.

Theorem 2 (Existence of equilibrium, Eaves (1985)) For a Cobb-Douglas economy the following assertions are equivalent:

(a) exchange equilibrium exists,

(b) economy has symmetric access,

(b) its corresponding matrix A from (3) is completely reducible.

\footnotetext{
${ }^{3}$ In particular, the endowments have to be reordered and written in accordance with the commodities in submarkets as $\omega=\left(\omega_{r}, r=1, \ldots, R\right)$ and $\omega_{i}=\left(\omega_{i r}, r=1, \ldots, R\right)$.
} 


\section{Tâtonnement for Cobb-Douglas economies}

We consider the following tâtonnements to compute equilibrium prices:

$$
\begin{gathered}
p_{k+1}:=\frac{x\left(p_{k}\right) \circ p_{k}}{\omega}, \quad \text { with } p_{0} \omega=1, \quad p_{0}>0 . \\
\bar{p}_{k+1}:=\frac{k+1}{k+2}\left(\frac{x\left(\bar{p}_{k}\right) \circ \bar{p}_{k}}{\omega}\right)+\frac{1}{k+2} \bar{p}_{0}, \quad \text { with } \bar{p}_{0} \omega=1, \quad \bar{p}_{0}>0 .
\end{gathered}
$$

We recall that $x\left(p_{k}\right) \circ p_{k}$ is the vector with components

$$
\left(x^{(j)}\left(p_{k}\right) p_{k}^{(j)}, \quad j=1, \ldots, n\right) .
$$

Both tâtonnements $\mathrm{T} 1$ and $\mathrm{T} 2$ can be expressed in terms of the matrix $A$, since

$$
A p=\frac{x(p) \circ p}{\omega} .
$$

We have

$$
p_{k+1}=A p_{k} \quad \text { and } \quad \bar{p}_{k+1}=\frac{k+1}{k+2} A \bar{p}_{k}+\frac{1}{k+2} \bar{p}_{0} .
$$

The proposed tâtonnements are power methods for solving the eigenvector problem

$$
A p=p \quad \text { with } p \omega=1, \quad p>0,
$$

where $\omega^{T} A=\omega^{T}$ and $w>0$. Note that the solutions of this eigenvalue problem coincide with the equilibrium prices for Cobb-Douglas economies. Moreover, the eigenvalue problem is solvable in case of symmetric access.

Now, we give an economic interpretation of $\mathrm{T} 1$ and $\mathrm{T} 2$. Let us rewrite $\mathrm{T} 1$ as

$$
p_{k+1}-p_{k}=\frac{\left(x\left(p_{k}\right)-\omega\right) \circ p_{k}}{\omega} .
$$

Recalling the formula for the excess demand $z(p)=x(p)-w$, we get

$$
p_{k+1}-p_{k}=\frac{z\left(p_{k}\right) \circ p_{k}}{\omega},
$$

which shows that the change of prices in $\mathrm{T} 1$ is proportional to the value of excess demand measured w.r.t. old prices. Due to (4), we have for T2:

$$
\bar{p}_{k}=\frac{1}{k+1} \sum_{l=0}^{k} p_{l},
$$

the average prices from $\mathrm{T} 1$.

Lemma 1 states that the tâtonnements T1 and T2 are well-defined. The proof is relegated to the Appendix.

Lemma 1 Tâtonnements T1 and T2 are well defined, i.e.

$$
p_{k}, \bar{p}_{k} \in \Delta_{1}(w) \text { for all } k=0,1, \ldots,
$$

where

$$
\Delta_{1}(w):=\left\{p \in \mathbb{R}^{n} \mid p \omega=1, \quad p>0\right\} .
$$


Proof: See Appendix.

Theorem 3 clarifies the convergence properties of tâtonnements T1 and T2 when there is symmetric access.

Theorem 3 (Convergence I) Let a Cobb-Douglas economy have symmetric access. Then,

(1) prices $p_{k}$ from tâtonnement $T 1$ may diverge,

(2) prices $\bar{p}_{k}$ from tâtonnement T2 converge to an equilibrium, but in general not faster than sublinearly.

Proof: See Appendix.

Theorem 3 shows that we need an additional assumption in order to obtain faster convergence for tâtonnements $\mathrm{T} 1$ and T2. For that, we use the decomposition of the Cobb-Douglas economy into submarkets $N_{r}, r=1, \ldots, R$, from above. Recall that the endowments are reordered in accordance with the commodities in submarkets as

$$
\omega=\left(\omega_{r}, r=1, \ldots, R\right), \quad \omega_{i}=\left(\omega_{i r}, r=1, \ldots, R\right), \quad i=1, \ldots, m .
$$

Definition 3 (Pairwise connectivity) The $r$-th submarket is said to be pairwise connected if for all pairs of its commodities $j, j^{\prime} \in N_{r}$ there are consumers $i$ and $i^{\prime}$ such that consumer $i$ possesses commodity $j$, consumer $i^{\prime}$ possesses commodity $j^{\prime}$, and they both desire some common commodity $\ell \in N_{r}$ from this submarket, i.e.

$$
\omega_{i r}^{(j)}, \omega_{i^{\prime} r}^{\left(j^{\prime}\right)}>0, \quad \alpha_{i \ell}, \alpha_{i^{\prime} \ell}>0 .
$$

A Cobb-Douglas economy is said to be pairwise connected if each of its submarkets is pairwise connected.

In terms of submarket matrices the pairwise connectivity of a Cobb-Douglas economy equivalently reads

$$
A_{r}^{T} A_{r}>0 \text { for all } r=1, \ldots, R
$$

We define the measure of pairwise connectivity for the $r$-th submarket as

$$
\pi_{r}=\pi\left(A_{r}, \omega_{r}\right):=\min _{j, j^{\prime} \in N_{r}} \sum_{\ell \in N_{r}} \omega_{r}^{(\ell)} \min \left\{\frac{1}{\omega_{r}^{(j)}}\left(A_{r} e_{j}\right)^{(\ell)}, \frac{1}{\omega_{r}^{\left(j^{\prime}\right)}}\left(A_{r} e_{j^{\prime}}\right)^{(\ell)}\right\},
$$

where $e_{j}, e_{j^{\prime}}$ are $j$-th and $j^{\prime}$-th coordinate vectors in $\mathbb{R}^{n_{r}}$, respectively. The measure of pairwise connectivity for the Cobb-Douglas economy is

$$
\pi:=\min _{1 \leq r \leq R} \pi_{r}
$$

Note that the Cobb-Douglas economy is pairwise connected if and only if $\pi>0$. Moreover,

$$
\begin{aligned}
\pi_{r} & =\min _{j, j^{\prime} \in N_{r}} \sum_{\ell \in N_{r}} \omega_{r}^{(\ell)} \min \left\{\frac{1}{\omega_{r}^{(j)}}\left(A_{r} e_{j}\right)^{(\ell)}, \frac{1}{\omega_{r}^{\left(j^{\prime}\right)}}\left(A_{r} e_{j^{\prime}}\right)^{(\ell)}\right\} \\
& \leq \min _{j, j^{\prime} \in N_{r}} \min \left\{\frac{1}{\omega_{r}^{(j)}} \sum_{\ell \in N_{r}} \omega_{r}^{(\ell)}\left(A_{r} e_{j}\right)^{(\ell)}, \frac{1}{\omega_{r}^{\left(j^{\prime}\right)}} \sum_{\ell \in N_{r}} \omega_{r}^{(\ell)}\left(A_{r} e_{j^{\prime}}\right)^{(\ell)}\right\} \\
& =\min _{j, j^{\prime} \in N_{r}} \min \left\{\frac{1}{\omega_{r}^{(j)}} \omega_{r}^{(j)}, \frac{1}{\omega_{r}^{\left(j^{\prime}\right)}} \omega_{r}^{\left(j^{\prime}\right)}\right\}=1,
\end{aligned}
$$


shows that $\pi \leq 1$.

The following result is crucial for the convergence analysis of tâtonnements T1 and T2 in the presence of pairwise connectivity. For that, we use the following scaled 1-norm on $\mathbb{R}^{n}$ :

$$
\|x\|_{v}=\sum_{j=1}^{n} v^{(j)}\left|x^{(j)}\right|
$$

for given vector $v=\left(v^{(1)}, \ldots, v^{(n)}\right)^{T}>0$.

Lemma 2 (Seminorm inequality, Nesterov and Nemirovski (2015)) Let $B \geq 0$ be an $n \times n$ matrix with $v^{T} B=v^{T}$ for a vector $v>0$. Then, for all $h \in \mathbb{R}^{n}$ with $v h=0$ it holds:

$$
\|B h\|_{v} \leq(1-\pi(B, v))\|h\|_{v},
$$

where

$$
\pi(B, v)=\min _{1 \leq j, j^{\prime} \leq n} \sum_{\ell=1}^{n} v^{(\ell)} \min \left\{\frac{1}{v^{(j)}}\left(B e_{j}\right)^{(\ell)}, \frac{1}{v\left(j^{\prime}\right)}\left(B e_{j^{\prime}}\right)^{(\ell)}\right\} \in[0,1] .
$$

Based on Lemma 2, we obtain convergence rates for tâtonnements $\mathrm{T} 1$ and T2 under pairwise connectivity.

Theorem 4 (Convergence II) Let a Cobb-Douglas economy have symmetric access and be pairwise connected. Then,

(1) prices $p_{k}$ from tâtonnement $T 1$ converge towards an equilibrium $p^{*}$ with linear rate

$$
\left\|p_{k}-p^{*}\right\|_{\omega} \leq(1-\pi)^{k}\left\|p_{0}-p^{*}\right\|_{\omega}, \quad k=0,1, \ldots,
$$

(2) prices $\bar{p}_{k}$ from tâtonnement T2 converge towards an equilibrium $p^{*}$ with sublinear rate

$$
\left\|\bar{p}_{k}-p^{*}\right\|_{\omega} \leq \frac{1-(1-\pi)^{k+1}}{(k+1) \pi}\left\|\bar{p}_{0}-p^{*}\right\|_{\omega}, \quad k=0,1, \ldots
$$

Proof: See Appendix.

Corollary 1 (Uniqueness of equilibrium) Let a Cobb-Douglas economy have symmetric access, no submarkets, and be pairwise connected. ${ }^{4}$ Then, it has a unique equilibrium price in $\Delta_{1}(w)$.

Proof: See Appendix.

Now, we state sufficient conditions for pairwise connectivity.

Definition 4 (Global connectivity) The $r$-th submarket is said to be globally connected if there exists a commodity $j \in N_{r}$ such that for all submarket commodities $j^{\prime} \in N_{r}$ there exists a consumer $i$ who possesses commodity $j^{\prime}$ and desires commodity $j$, i.e.

$$
\omega_{i r}^{\left(j^{\prime}\right)}>0, \quad \alpha_{i j}>0 .
$$

A Cobb-Douglas economy is said to be globally connected if each of its submarkets is globally connected.

\footnotetext{
${ }^{4}$ Cobb-Douglas economies with these properties will later be called regular. See Definition 6 .
} 
In terms of submarket matrices the global connectivity of a Cobb-Douglas economy equivalently says that each matrix $A_{r}, r=1, \ldots, R$, has at least one positive row. We define the measure of global connectivity for the $r$-th submarket as

$$
\gamma_{r}=\gamma\left(A_{r}, \omega_{r}\right):=\sum_{\ell=1}^{N_{r}} \omega_{r}^{(\ell)} \min _{j \in N_{r}} \frac{1}{\omega_{r}^{(j)}}\left(A_{r} e_{j}\right)^{(\ell)} .
$$

The measure of pairwise connectivity for the Cobb-Douglas economy is

$$
\gamma:=\min _{1 \leq r \leq R} \gamma_{r}
$$

Note that the Cobb-Douglas economy is globally connected if and only if $\gamma>0$.

Definition 5 (Full connectivity) The $r$-th submarket is said to be fully connected if for all pairs of its commodities $j, j^{\prime} \in N_{r}$ there is a consumer $i$ who possesses commodity $j^{\prime}$ and desires commodity $j \in N_{r}$, i.e.

$$
\omega_{i r}^{\left(j^{\prime}\right)}>0, \quad \alpha_{i j}>0
$$

A Cobb-Douglas economy is said to be fully connected if each of its submarkets is fully connected.

In terms of submarket matrices the full connectivity of a Cobb-Douglas economy equivalently says that each matrix $A_{r}, r=1, \ldots, R$, has only positive elements. We define the measure of full connectivity for the $r$-th submarket as

$$
\phi_{r}=\phi\left(A_{r}, \omega_{r}\right):=\sum_{\ell \in N_{r}} \omega_{r}^{(\ell)} \min _{j, j^{\prime} \in N_{r}} \frac{1}{\omega_{r}^{(j)}}\left(A_{r} e_{j}\right)^{\left(j^{\prime}\right)}
$$

The measure of full connectivity for the Cobb-Douglas economy is

$$
\phi:=\min _{1 \leq r \leq R} \phi_{r}
$$

Note that the Cobb-Douglas economy is fully connected if and only if $\phi>0$.

Lemma 3 (Connectivity relations) The following (weak) inequalities hold between the measures of connectivity:

$$
\pi_{r} \geq \gamma_{r} \geq \phi_{r}, \quad r=1, \ldots, R \text { and } \pi \geq \gamma \geq \phi .
$$

In particular, if the $r$-th submarket of a Cobb-Douglas economy is fully connected then it is also globally connected. If a Cobb-Douglas economy is fully connected then it is also globally connected. If the $r$-th submarket of a Cobb-Douglas economy is globally connected then it is also pairwise connected. If a Cobb-Douglas economy is globally connected then it is also pairwise connected. 
Proof: We have

$$
\begin{aligned}
\pi_{r} & =\min _{j, j^{\prime} \in N_{r}} \sum_{\ell \in N_{r}} \omega_{r}^{(\ell)} \min \left\{\frac{1}{\omega_{r}^{(j)}}\left(A_{r} e_{j}\right)^{(\ell)}, \frac{1}{\omega_{r}^{\left(j^{\prime}\right)}}\left(A_{r} e_{j^{\prime}}\right)^{(\ell)}\right\} \\
& \geq \min _{j, j^{\prime} \in N_{r}} \sum_{\ell \in N_{r}} \omega_{r}^{(\ell)} \min _{j \in N_{r}} \frac{1}{\omega_{r}^{(j)}}\left(A_{r} e_{j}\right)^{(\ell)}=\gamma_{r} \\
& =\sum_{\ell \in N_{r}} \omega_{r}^{(\ell)} \min _{j \in N_{r}} \frac{1}{\omega_{r}^{(j)}}\left(A_{r} e_{j}\right)^{(\ell)} \geq \sum_{\ell \in N_{r}} \omega_{r}^{(\ell)} \min _{j, \ell \in N_{r}} \frac{1}{\omega_{r}^{(j)}}\left(A_{r} e_{j}\right)^{(\ell)}=\phi_{r} .
\end{aligned}
$$

The conclusion follows immediately.

Example 1 (Capital and labor) We consider a Cobb-Douglas economy with $n$ commodities, namely, consumption commodities $1, \ldots, n-2$, capital $n-1$, and labor $n$. Let producers offer consumption commodities $1, \ldots, n-2$ in exchange for capital and labor; capitalists offer capital by using labor and desire consumption commodities; workers supply labor by using capital and desire consumption commodities. Then, the corresponding matrix of this capitalistic Cobb-Douglas economy is

$$
A=\left(\begin{array}{ccccc}
0 & \ldots & 0 & \star & \star \\
\ldots & \ldots & \ldots & \ldots & \ldots \\
0 & \ldots & 0 & \star & \star \\
\star & \ldots & \star & 0 & \star \\
\star & \ldots & \star & \star & 0
\end{array}\right),
$$

where $\star$ indicates nonzero elements. We immediately see that $A$ is irreducible, $A^{T} A>0$, but $A$ does not have positive rows. Hence, the capitalistic Cobb-Douglas economy has symmetric access, it is pairwise, but neither globally nor fully connected. Note that in absence of capital or labor, the corresponding Cobb-Douglas economy may not be pairwise connected at all.

Example 2 (Money / Basic need) We consider a Cobb-Douglas economy with $n-1$ commodities and a special $n$-th commodity, which is desired by all consumers. This special commodity may play the role of money or be required to satisfy some basic need of every consumer. Due to Assumption 1, the corresponding matrix of this "monetary" Cobb-Douglas economy has positive $n$-th row. This economy is globally connected and, hence, also pairwise connected.

Example 3 (Taxation) We consider a Cobb-Douglas economy with $n$ commodities and a special consumer, who possesses some amount of every commodity. This special consumer may play the role of the state collecting taxes from other consumers. Due to Assumption 2, the special consumer desires some commodity $j$. Then, the corresponding matrix of the regulated Cobb-Douglas economy has a positive $j$-th row. This regulated Cobb-Douglas economy is globally connected and, hence, also pairwise connected. 


\section{Regularity of Cobb-Douglas economies}

Let the number of consumers $m$ and exchange commodities $n$ be fixed. We consider the set of Cobb-Douglas economies with overall endowments $\omega>0$ fulfilling Assumptions 1 and 2 :

$$
\mathcal{E}:=\left\{\begin{array}{l|l}
(\alpha, \mathcal{W})=\left(\alpha_{i}, \omega_{i}\right)_{i=1}^{m} \in[0,1]^{n m} \times \mathbb{R}_{+}^{n m} & \begin{array}{l}
\sum_{i=1}^{m} \omega_{i}=\omega \\
\sum_{i=1}^{m} \alpha_{i j}>0 \text { for all } j=1, \ldots, n \\
\sum_{j=1}^{n} \alpha_{i j}=1 \text { for all } i=1, \ldots, m
\end{array}
\end{array}\right\} .
$$

Definition 6 (Regular Cobb-Douglas economy) A Cobb-Douglas economy $(\alpha, \mathcal{W}) \in$ $\mathcal{E}$ is called regular if it has symmetric access, no submarkets, and is pairwise connected.

Due to Theorem 4 and Corollary 1, tâtonnement T1 approximates the unique equilibrium of a regular Cobb-Douglas economy at the linear rate. We show that the subset of regular economies is open and dense in $\mathcal{E}$. This needs several auxiliary results of independent interest.

Lemma 4 (Stability of symmetric access) The subset of Cobb-Douglas economies having symmetric access and no submarkets is open in $\mathcal{E}$.

Proof: See Appendix.

Lemma 5 (Density of full connectivity) The subset of fully connected Cobb-Douglas economies having symmetric access and no submarkets is dense in $\mathcal{E}$.

Proof: See Appendix.

Theorem 5 (Genericity of regular Cobb-Douglas economies) The subset of regular Cobb-Douglas economies is open and dense in $\mathcal{E}$.

Proof: See Appendix.

Analogously to the proof of Theorem 5, it can be shown that the following subsets of Cobb-Douglas economies from $\mathcal{E}$ are also open and dense:

- with symmetric access and no submarkets;

- with symmetric access, no submarkets, and globally connected;

- with symmetric access, no submarkets, and fully connected.

Note that the absence of submarkets in Definition 6 of regular Cobb-Douglas economies is essential for their stability.

Proposition 1 (Instability of Cobb-Douglas economies with submarkets) Let a Cobb-Douglas economy $(\alpha, \mathcal{W}) \in \mathcal{E}$ with submarkets have symmetric access. Then, there exists a Cobb-Douglas economy without symmetric access arbitrarily close to $(\alpha, \mathcal{W})$.

Proof: See Appendix.

It follows from Proposition 1 that the globalization of a Cobb-Douglas economy through submarket merges may induce a pathological Cobb-Douglas economy without an exchange equilibrium. 


\section{References}

Arrow, K., Block, H., and Hurwicz, L. (1959). On the stability of the competitive equilibrium. Econometrica, 27:82-109.

Arrow, K. and Hurwicz, L. (1958). On the stability of the competitive equilibrium. Econometrica, 26:522-552.

Bonnisseau, J.-M. (2003). Regular economies with non-ordered preferences. Journal of Mathematical Economics, 39:153-174.

Bonnisseau, J.-M., Florig, M., and Jofré, A. (2001). Continuity and uniqueness of equilibria for linear exchange economies. Journal of Optimization Theory and Applications, 109:237-263.

Debreu, G. (1970). Economies with a finite set of equilibria. Econometrica, 38:387-392.

Du, Y., Lehrer, E., and Pauzner, A. (2015). Competitive economy as a ranking device over networks. Games and Economic Behavior, 91:1-13.

Eaves, B. C. (1976). A finite algorithm for the linear exchange model. Journal of Mathematical Economics, 3:197-203.

Eaves, B. C. (1985). Finite solution of pure trade markets with Cobb-Douglas utilities. Mathematical Programming, 23:226-239.

Mas-Collel, A. (1985). The Theory of General Economic Equilibrium: a Differentiable Approach. Cambridge University Press, Cambridge.

Nesterov, Y. and Nemirovski, A. (2015). Finding the stationary states of Markov chains by iterative methods. Applied Mathematics and Computation, 255:58-65.

Scarf, H. (1960). Some examples of global instability of the competitive equilibrium. International Economic Review, 1:157-172.

Scarf, H. (1967). The approximation of fixed points of a continuous mapping. SIAM Journal of Applied Mathematics, 15:1328-1343.

Scarf, H. (1973). The Computation of Economic Equilibria. Yale University Press, New Haven.

Schaefer, H. H. (1974). Banach Lattices and Positive Operators. Springer, BerlinHeidelberg.

\section{Appendix}

Proof of Lemma 1: If $p>0$, then aggregate demand can be written as

$$
x^{(j)}(p)=\sum_{i=1}^{m} \frac{\alpha_{i j}}{p^{(j)}} p \omega_{i}, \quad j=1, \ldots, n .
$$

Due to Assumption 1, for every commodity $j$ there exists a consumer $i$ with $\alpha_{i j}>0$. Note that since $\omega_{i} \neq 0$ for all $i, p \omega_{i}>0$. Overall, $x(p)>0$. This argument allows to prove by induction that $p_{k}>0$ and $\bar{p}_{k}>0$ for all $k=0,1, \ldots$ 
Furthermore, due to $\omega^{T} A=\omega^{T}$, the following holds:

$$
p_{k+1} \omega=A p_{k} \omega=p_{k} A^{T} \omega=p_{k} \omega,
$$

and

$$
\begin{aligned}
p_{k+1} \omega & =\left(\frac{k+1}{k+2} A \bar{p}_{k}+\frac{1}{k+2} \bar{p}_{0}\right) \omega \\
& =\frac{k+1}{k+2} \bar{p}_{k} A^{T} \omega+\frac{1}{k+2} \bar{p}_{0} \omega=\frac{k+1}{k+2} \bar{p}_{k} \omega+\frac{1}{k+2} .
\end{aligned}
$$

By induction, we also obtain that $p_{k} \omega=\bar{p}_{k} \omega=1$ for all $k=0,1, \ldots$

Proof of Theorem 3: (1) We define a Cobb-Douglas economy with $n$ commodities and $m=n$ consumers. The $i$-th consumer's has endowment $\omega_{i}=e_{i}$ and consumption elasticities

$$
\alpha_{i j}= \begin{cases}1 & \text { if } j=\psi(i) \\ 0 & \text { else }\end{cases}
$$

where $\psi$ is a cyclic permutation of $\{1, \ldots, n\}$. Note that this Cobb-Douglas economy satisfies Assumptions 1 and 2. It has symmetric access due to Theorem 1, since there is a vector of positive equilibrium prices $\frac{1}{n} e$. Further, we see that

$$
A=\left(\begin{array}{c}
e_{\psi^{-1}(1)}^{T} \\
\vdots \\
e_{\psi^{-1}(n)}^{T}
\end{array}\right)
$$

is a cyclic permutation matrix. For tâtonnement T1 we have

$$
p_{k}=A p_{k-1}=A^{k} p^{0}=\left(p_{0}^{\left(\psi^{-k}(1)\right)}, \ldots, p_{0}^{\left(\psi^{-k}(n)\right)}\right) .
$$

Obviously, $p^{k}$ oscillates unless $p_{0}=\left(\frac{1}{n}, \ldots, \frac{1}{n}\right)$, since $\psi^{-n}=\psi$.

(2) Tâtonnement T2 can be written as

$$
\bar{p}_{k}=\frac{k}{k+1} A \bar{p}_{k-1}+\frac{1}{k+1} \bar{p}_{0}=\frac{1}{k+1} \sum_{l=0}^{k} A^{l} \bar{p}^{0} .
$$

Due to Theorem 1, the property of symmetric access implies that $\frac{1}{k+1} \sum_{l=0}^{k} A^{l} \rightarrow P$, $k \rightarrow \infty$, where the matrix $P$ cannot have a zero row. Because of $A P=P$, each column of $P$ is an eigenvector of $A$ for the eigenvalue 1 . Hence, $p^{*}:=P \bar{p}_{0}$ is a positive eigenvector of $A$ for all $\bar{p}_{0}>0$, i.e. an equilibrium price because of Assumptions 1 and 2 .

Now, let us estimate the convergence rate of the tâtonnement T2 for the permutation economy from (1). For $k=\ln$ with $l=1,2, \ldots$ we compute

$$
\bar{p}_{k}=\frac{l}{k+1} e+\frac{1}{k+1} \bar{p}_{0}
$$

With the unique equilibrium price $p^{*}=\frac{1}{n} e$ we obtain

$$
\left\|\bar{p}_{k}-p^{*}\right\|_{e}=\frac{1}{k+1}\left\|\bar{p}_{0}-p^{*}\right\|_{e} .
$$


If $p_{0} \rightarrow e_{j}$ with $j \in\{1, \ldots, n\}$, then $\left\|\bar{p}_{0}-p^{*}\right\|_{e} \rightarrow \frac{2(n-2)}{n}$. Hence, the convergence $\bar{p}_{k} \rightarrow p^{*}$ is not faster than sublinear w.r.t. the number of steps $k$.

Proof of Theorem 4: Tâtonnements T1 and T2 can be equivalently written for each submarket $r=1, \ldots, R$ as:

$$
\begin{gathered}
p_{k+1 r}=\frac{x_{r}\left(p_{k}\right) \circ p_{k r}}{\omega_{r}}=A_{r} p_{k r}, \\
\bar{p}_{k+1 r}=\frac{k+1}{k+2}\left(\frac{x_{r}\left(\bar{p}_{k}\right) \circ \bar{p}_{k r}}{\omega_{r}}\right)+\frac{1}{k+2} \bar{p}_{0 r},
\end{gathered}
$$

where

$$
p_{k}=\left(p_{k 1}, \ldots, p_{k R}\right), \quad x\left(p_{k}\right)=\left(x_{1}\left(p_{k}\right), \ldots, x_{R}\left(p_{k}\right)\right), \quad \omega=\left(\omega_{1}, \ldots, \omega_{R}\right) .
$$

Note first that equality $\omega_{r}^{T} A_{r}=\omega_{r}^{T}$ holds for each $n_{r} \times n_{r}$ matrix $A_{r} \geq 0$. For $p_{0}=$ $\left(p_{01}, \ldots, p_{0 R}\right)>0$ we also define

$$
t_{r}:=p_{0 r} \omega_{r}>0, \quad r=1, \ldots, R
$$

as well as

$$
\Delta_{t_{r}}\left(\omega_{r}\right):=\left\{p_{r} \in \mathbb{R}^{n_{r}} \mid p_{r} \omega_{r}=t_{r}, \quad p_{r}>0\right\} .
$$

Clearly, $\sum_{r=1}^{R} t_{r}=1$, and as in Lemma 1 it is straightforward to show that

$$
p_{k r}, \bar{p}_{k r} \in \Delta_{t_{r}}\left(\omega_{r}\right) \text { for all } r=1, \ldots, R \text { and } k=0,1, \ldots
$$

Recall that the endowments of consumer $i$ are rewritten as

$$
\omega_{i}=\left(\omega_{i 1}, \ldots, \omega_{i R}\right)
$$

according to the submarket commodities.

(1) Let $p^{*}=\left(p_{1}^{*}, \ldots, p_{R}^{*}\right)>0$ be an equilibrium price. By scaling all prices in the block $p_{r}^{*}$ with $\frac{t_{r}}{\omega_{r} p_{r}^{*}}$, we may achieve w.l.o.g. that $p_{r}^{*} \in \Delta_{t_{r}}\left(\omega_{r}\right), r=1, \ldots, R$. The following inequality holds:

$$
\begin{gathered}
\left\|p_{k}-p^{*}\right\|_{\omega}=\left\|A\left(p_{k-1}-p^{*}\right)\right\|_{\omega} \\
=\sum_{r=1}^{R}\left\|A_{r}\left(p_{k-1 r}-p_{r}^{*}\right)\right\|_{\omega_{r}} \stackrel{\text { Lemma } 2}{\leq} \sum_{r=1}^{R}\left(1-\pi_{r}\right)\left\|p_{k-1 r}-p_{r}^{*}\right\|_{\omega_{r}} \\
\leq(1-\pi) \sum_{r=1}^{R}\left\|p_{k-1 r}-p_{r}^{*}\right\|_{\omega_{r}}=(1-\pi)\left\|p_{k-1}-p^{*}\right\|_{\omega} .
\end{gathered}
$$

Lemma 2 is applicable, since by using $p_{k r} \in \Delta_{t_{r}}\left(\omega_{r}\right)$ we have

$$
\omega_{r}\left(p_{k r}-p_{r}^{*}\right)=\omega_{r}, p_{k r}-\omega_{r} p_{r}^{*}=t_{r}-t_{r}=0 .
$$

Moreover, $\omega_{r}^{T} A_{r}=\omega_{r}^{T}$ for $r=1, \ldots, R$. Due to the property of symmetric access, $\pi>0$, hence, $p_{k}$ converges towards the equilibrium price $p^{*}$. 
(2) The following inequality holds:

$$
\begin{gathered}
\left\|\bar{p}_{k}-p^{*}\right\|_{\omega}=\left\|\frac{1}{k+1} \sum_{l=0}^{k} p_{l}-p^{*}\right\|_{\omega} \leq \frac{1}{k+1} \sum_{l=0}^{k}\left\|p_{l}-p^{*}\right\|_{\omega} \\
\stackrel{(1)}{\leq} \frac{1}{k+1} \sum_{l=0}^{k}(1-\pi)^{l}\left\|p_{0}-p^{*}\right\|_{\omega}=\frac{1-(1-\pi)^{k+1}}{(k+1) \pi}\left\|\bar{p}_{0}-p^{*}\right\|_{\omega} .
\end{gathered}
$$

Proof of Corollary 1: We apply the proof of Theorem 4 for $R=1$. Then, for any equilibrium price $p^{*} \in \Delta_{1}(\omega), p_{k} \rightarrow p^{*}$ for $k \rightarrow \infty$. Hence, $p^{*}$ is unique as the limit of $p_{k}$ 's.

Proof of Lemma 4: Let $(\alpha, \mathcal{W}) \in \mathcal{E}$ be a Cobb-Douglas economy having symmetric access and no submarkets. It is equivalent to the irreducibility of the corresponding matrix $A$ from (3). Assume that contrarily to the assertion there exists a sequence of CobbDouglas economies $\left(\alpha_{\varepsilon}, \mathcal{W}_{\varepsilon}\right) \in \mathcal{E}$ with not irreducible matrices $A_{\varepsilon}$ which approximate $(\alpha, \mathcal{W})$ as $\varepsilon \rightarrow 0$. Clearly, $A_{\varepsilon} \rightarrow A$ for $\varepsilon \rightarrow 0$, and there exists permutation matrices $P_{\varepsilon}$ such that

$$
P_{\varepsilon}^{-1} A_{\varepsilon} P_{\varepsilon}=\left(\begin{array}{cc}
C_{\varepsilon} & 0 \\
D_{\varepsilon} & E_{\varepsilon}
\end{array}\right)
$$

where $C_{\varepsilon}$ and $E_{\varepsilon}$ are square matrices of dimensions smaller than $n$. Taking a subsequence of $\left(\alpha_{\varepsilon}, \mathcal{W}_{\varepsilon}\right)$ if needed, we obtain

$$
P^{-1} A_{\varepsilon} P=\left(\begin{array}{cc}
C_{\varepsilon} & 0 \\
D_{\varepsilon} & E_{\varepsilon}
\end{array}\right)
$$

with a fixed permutation matrix $P$ and the same sizes of $C_{\varepsilon}$ and $D_{\varepsilon}$, respectively, for all $\varepsilon$. Letting $\varepsilon \rightarrow 0$ we get:

$$
P^{-1} A P=\left(\begin{array}{cc}
C & 0 \\
D & E
\end{array}\right)
$$

with square matrices $C$ and $D$. Hence, $A$ is not irreducible, a contradiction.

Proof of Lemma 5: Let $(\alpha, \mathcal{W}) \in \mathcal{E}$ be a Cobb-Douglas economy. By arbitrarily small perturbations of consumers' tastes $\alpha$ and endowments $\mathcal{W}$, we may obtain a CobbDouglas economy $(\widetilde{\alpha}, \widetilde{\mathcal{W}}) \in \mathcal{E}$ whose corresponding matrix $\widetilde{A}$ has only positive elements and is irreducible. For that, let each consumer desire and posses just an arbitrarily small quantity of every commodity. Hence, the corresponding Cobb-Douglas economy $(\widetilde{\alpha}, \widetilde{\mathcal{W}})$ has symmetric access and no submarkets. Moreover, it is also fully connected.

Proof of Theorem 5: Due to Lemma 4, the subset of Cobb-Douglas economies having symmetric access and no submarkets is open in $\mathcal{E}$. Those pairwise connected economies 
$(\alpha, \mathcal{W}) \in \mathcal{E}$ are characterized by the condition $\pi(A, \omega)>0$, where

$$
\pi(A, \omega)=\min _{1 \leq j, j^{\prime} \leq n} \sum_{\ell=1}^{n} \omega^{(\ell)} \min \left\{\frac{1}{\omega^{(j)}}\left(A e_{j}\right)^{(\ell)}, \frac{1}{\omega^{\left(j^{\prime}\right)}}\left(A e_{j^{\prime}}\right)^{(\ell)}\right\} .
$$

Note that the dependence of $A$ on $(\alpha, \mathcal{W})$ is continuous, as well as the dependence of $\pi(A, \omega)$ on the elements of $A$. Hence, pairwise connected economies remain stable under sufficiently small perturbations of consumers' tastes and endowments $(\alpha, \mathcal{W})$. Overall, the subset of regular Cobb-Douglas economies is open. Further, Lemma 3 states that the full connectivity implies pairwise connectivity of Cobb-Douglas economies. Hence, density of regular Cobb-Douglas economies is due to Lemma 5.

Proof of Proposition 1: Let a Cobb-Douglas economy $(\alpha, \mathcal{W}) \in \mathcal{E}$ with submarkets have symmetric access. Its corresponding matrix $A$ is completely reducible and, hence, up to a permutation of commodities can be written as

$$
A=\left(\begin{array}{cccc}
A_{1} & 0 & \ldots & 0 \\
0 & A_{2} & \ldots & 0 \\
\ldots & \ldots & \ldots & \ldots \\
0 & 0 & \ldots & A_{R}
\end{array}\right)
$$

where the number of submarkets $R \geq 2$, and $A_{r}, r=1, \ldots, R$ are irreducible square $n_{r} \times n_{r}$ matrices. By arbitrarily small perturbations of consumers' endowments $\mathcal{W}$, we may obtain a Cobb-Douglas economy $(\bar{\alpha}, \overline{\mathcal{W}}) \in \mathcal{E}$ whose corresponding matrix is

$$
\bar{A}=\left(\begin{array}{cccc}
\bar{A}_{1} & 0 & \ldots & 0 \\
D & A_{2} & \ldots & 0 \\
\ldots & \ldots & \ldots & \ldots \\
0 & 0 & \ldots & A_{R}
\end{array}\right)
$$

Here, $D$ is an $n_{2} \times n_{1}$ matrix with only positive elements, and $\bar{A}_{1}$ is an $n_{1} \times n_{1}$ irreducible matrix. Indeed, for every commodity $j \in N_{2}$ from the second submarket we may find a consumer $i$ who desires this commodity, i.e. $\alpha_{i j}>0$. Further, let us marginally enlarge the $i$-th consumer's endowments $\omega_{i}^{(\ell)}$ of commodities $\ell \in N_{1}$ from the first submarket. In order to balance the overall endowment, we reduce the endowments of those consumers who hold commodities $\ell \in N_{1}$ by an appropriate sufficiently small quantity. Note that this procedure only affects the first submarket's matrix $\bar{A}_{1}$ and induces an $n_{2} \times n_{1}$ matrix $D$ with only positive elements.

Now, we show that $\bar{A}$ is not completely reducible. Otherwise, due to Theorem 1 , there would exist a positive eigenvector $\bar{x}=\left(\bar{x}_{1}, \bar{x}_{2}, \ldots, x_{R}\right)^{T}>0$ of $\bar{A}$ for eigenvalue 1 . In particular,

$$
D \bar{x}_{1}+A_{2} \bar{x}_{2}=\bar{x}_{2} .
$$

Multiplying the latter equation by the vector of endowments $\omega_{2}>0$ corresponding to the commodities of the second submarket, we get

$$
\omega_{2} D \bar{x}_{1}+\omega_{2} A_{2} \bar{x}_{2}=\omega_{2} \bar{x}_{2} .
$$


Due to the fact that $\omega_{2}^{T} A_{2}=\omega_{2}^{T}$,

$$
D^{T} \omega_{2} \bar{x}_{1}=0 .
$$

Since $D^{T} \omega_{2}>0$, we obtain $\bar{x}_{1}=0$, a contradiction. Overall, $\bar{A}$ is not completely reducible, hence, the perturbed Cobb-Douglas economy $(\bar{\alpha}, \overline{\mathcal{W}})$ does not have symmetric access. 